Revisión narrativa o de literatura

\title{
¿ES LA REVISIÓN SISTEMÁTICA EL MEJOR ESTUDIO PARA TOMAR DECISIONES EN SALUD?
}

\author{
ARE SYSTEMATIC REVIEWS THE BEST STUDY FOR DECISION MAKING IN \\ HEALTH?
}

\section{Maria Soledad Kappes R.}

Enfermera Matrona

Mg. Ciencias Médicas

Profesor asistente

Escuela de Enfermería

Facultad de Ciencias para el Cuidado de la Salud

Universidad San Sebastián

Colaborador Cochrane Iberoamericano

\section{Cecilia Pacheco}

Grupo documentalistas clínicos

Centro Cochrane Chile

$\overline{\text { Artículo recibido el } 7 \text { de agosto de 2018. Aceptado en versión corregida el } 13 \text { de diciembre }}$ de 2018.

\section{RESUMEN}

Objetivo: Definir el mejor diseño de investigación para la práctica de enfermería. Metodología: se realiza una revisión narrativa con los términos MeSH: Alfabetización Informacional, Estudios Epidemiológicos, Medicina Basada en la Evidencia, Enfermería basada en evidencia en las bases de datos PUBMED, ProQuest, Ebsco, Ovid. Síntesis del contenido: Se realiza una síntesis de los diseños de investigación disponibles y su utilidad al momento de responder una pregunta de investigación. Conclusiones: La mejor evidencia disponible son las revisiones sistemáticas y el enfoque de investigación mixto. Su uso para práctica basada en evidencia asegura que podamos sustentar nuestros cuidados de manera eficiente y segura.

Palabras clave: Alfabetización Informacional, Estudios Epidemiológicos, Medicina Basada en la Evidencia, Enfermería basada en evidencia

\footnotetext{
ABSTRACT

Objective: Define the best research design for nursing practice. Methodology: a narrative review is carried out with the MeSH terms: Information Literacy, Epidemiological Studies, Evidence-Based Medicine, Evidence-Based Nursing in the PUBMED, ProQuest, Ebsco, Ovid databases. Synthesis of the content: A synthesis of the available research designs and Correspondencia e-mail: maria.kappes@uss.cl
} 
¿Es la revisión sistemática el mejor estudio...?

their usefulness when answering a research question is made. Conclusions: The best available evidence is the systematic reviews and the mixed research approach. Its use for evidencebased practice ensures that we can sustain our care efficiently and safely.

Keywords: Information Literacy, Epidemiological Studies, Evidence-Based Medicine, evidence based nursing

\section{http://dx.doi.org/10.7764/Horiz_Enferm.29.3.224-237}

\section{INTRODUCCIÓN}

Los profesionales de la salud nos vemos enfrentados a diario, a preguntas y dudas que surgen de la práctica clínica, es decir de nuestros pacientes. Localmente tenemos guías clínicas y consensos de expertos que pueden orientar nuestras decisiones y entregarnos el background del conocimiento necesario. Sin embargo, ¿podríamos afirmar que los consejos o cuidados que estamos entregando, son realmente los más válidos y eficaces? Probablemente no. La respuesta a esta interrogante ha sido analizada por investigadores, desde que la práctica clínica basada en evidencia (PBE) fue acuñada como término para definir la forma en que la evidencia científica es puesta al servicio de la salud ${ }^{(1)}$.

Entre otras, la Enfermería Basada en Evidencia (EBE) definida por Ingersoll (2000, en Morales, 2003: 38) como "la utilización consciente, explícita y juiciosa (crítica) de teoría derivada; la información basada en investigación en la toma de decisiones para el cuidado que se da a individuos o grupos de pacientes en consideración con las necesidades y preferencias individuales"(2). De estas definiciones, destacamos que la búsqueda de información debe ser acuciosa y consciente, pues desde allí enfrentaremos la práctica necesaria para el cuidado del paciente y las investigaciones derivadas, con resultados más eficientes y de mejor calidad.

En la búsqueda de respuestas para nuestras preguntas y dudas, el primer paso será consultar todos los estudios o artículos que aparecen en la infinidad de bases de datos existentes. Una tarea ardua $\mathrm{y}$ poco fructífera si no tenemos una metodología o un buen plan de ejecución previo $^{(3)}$.

Entonces, la búsqueda de la mejor evidencia científica disponible debiera ser el fundamento de los cuidados de enfermería. De esta forma, se establece la mejora no sólo la calidad de la atención, sino también su costo-efectividad ${ }^{(4)}$.

El propósito de esta revisión es determinar, dentro de los diseños epidemiológicos disponibles, cuál es la mejor contribución a la práctica de enfermería. Para ello, esta revisión explorará los tipos de diseños disponibles y la forma en que ellos contribuyen a la evidencia que puede sustentar la práctica de enfermería.

\section{Evidencia Disponible}

Comenzamos con la determinación del tipo de estudio que se necesita para rescatar la evidencia o pruebas necesarias, que puedan responder la pregunta 
Keppes M.S., Pacheco C.

planteada desde el ámbito en que se ha generado, pues no tienen la misma significación. Son diferentes las que se originan del intercambio de información médico-paciente, que las que se generan para lograr mayor conocimiento. Es así como se podrían diferenciar en: Preguntas Clínicas y Preguntas de Investigación $^{(5,6)}$. Ambas requieren del mismo y predeterminado proceso (Figura 1).

Elaboración de una pregunta de Investigación

Uno de los formatos más útiles para formular una pregunta de investigación es el formato $\operatorname{PICOT}^{(7)}$. Este formato que responde a una nemotecnia, resumen los aspectos esenciales que

Figura 1. Pregunta clínica y pregunta de investigación.

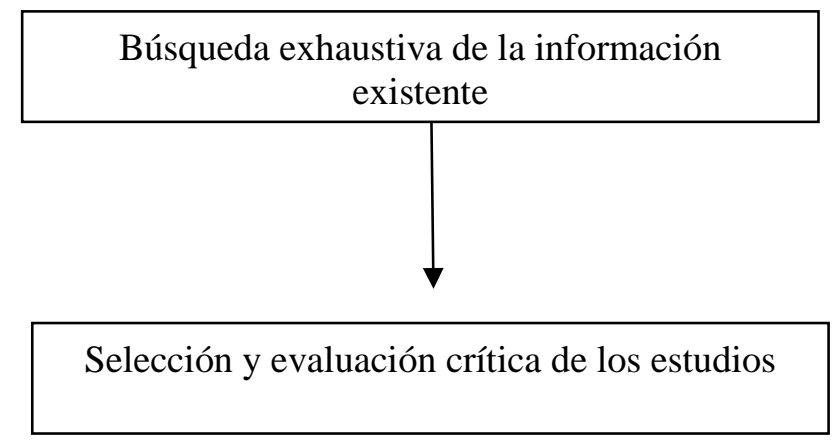

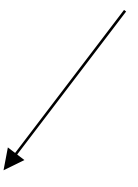

Práctica clínica /evidencia científica

Determinar beneficios y riesgos para el paciente desde información científicamente validada

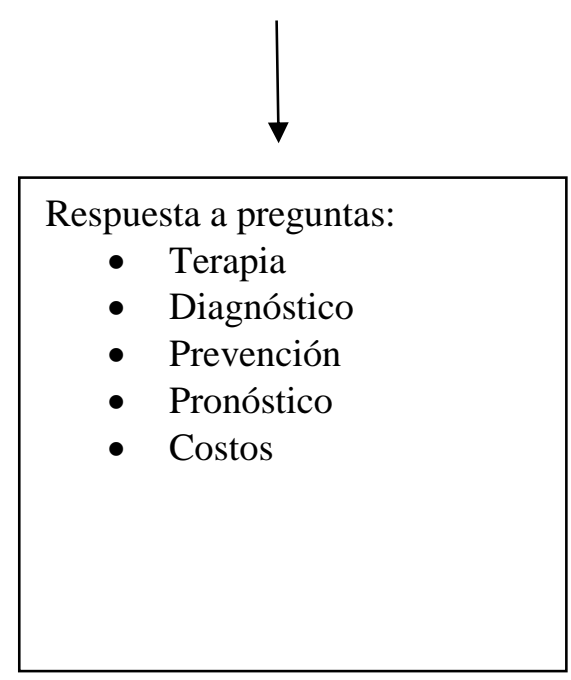

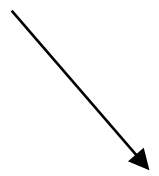

Investigación/evidencia científica

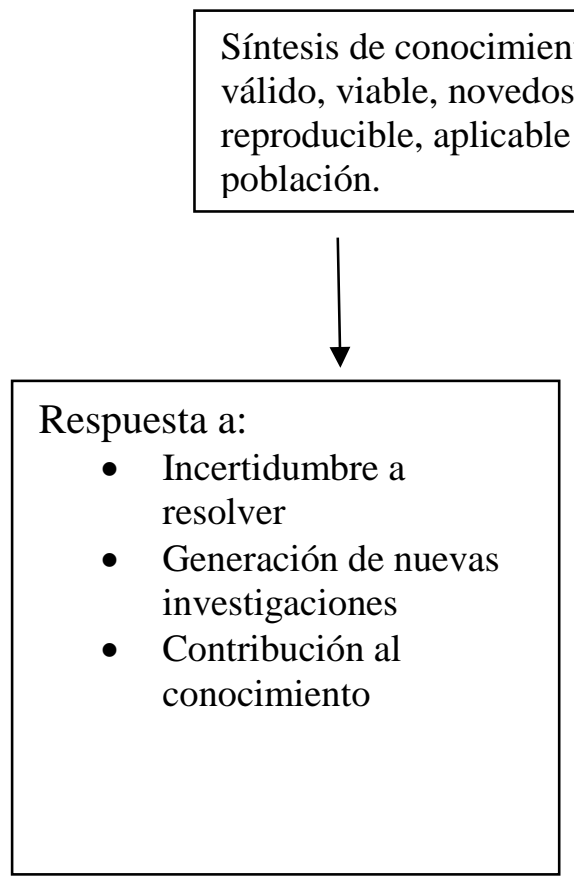



Keppes, M.S.

permiten centrar, en una pregunta, lo que queremos investigar.

P: Población

I: Intervención propuesta

C: comparación (o el "goldstandard" habitualmente usado con el que contrastamos la intervención)

O: Outcome o resultado
T: tempo

En la Figura 2 se muestran ejemplos de preguntas en formato PICOT.

\section{Según el enfoque de la Investigación}

Según el enfoque de la investigación, podemos clasificarlas en cuantitativa, cualitativa y mixta ${ }^{(8)}$.

Figura 2. Pregunta en formato PICOT.

\begin{tabular}{|c|c|c|}
\hline & Ejemplo 1 & Ejemplo 2 \\
\hline $\mathbf{P}$ & $\begin{array}{c}\text { Pacientes con accidente } \\
\text { cerebro vascular y secuela } \\
\text { motora }\end{array}$ & $\begin{array}{l}\text { Pacientes en ventilación } \\
\text { mecánica por más de } 72 \text { horas }\end{array}$ \\
\hline $\mathbf{I}$ & $\begin{array}{c}\text { Ejercicios pasivos cada } 4 \\
\text { horas }\end{array}$ & $\begin{array}{l}\text { Tubo endotraqueal con } \\
\text { aspiración subglótica }\end{array}$ \\
\hline $\mathbf{C}$ & $\begin{array}{c}\text { Ejerciticos pasivos cada } 8 \\
\text { horas }\end{array}$ & $\begin{array}{l}\text { Tubo endotraquealstandard y } \\
\text { aspiración de secreciones SOS }\end{array}$ \\
\hline $\mathbf{O}$ & $\begin{array}{c}\text { Prevención de atrofia } \\
\text { muscular }\end{array}$ & $\begin{array}{c}\text { Prevención de neumonía } \\
\text { nosocomial }\end{array}$ \\
\hline $\mathbf{T}$ & 1 año & $\begin{array}{l}1000 \text { días de uso de ventilación } \\
\text { mecánica }\end{array}$ \\
\hline Pregunta formulada & $\begin{array}{l}\text { ¿En pacientes con accidente } \\
\text { cerebro vascular y secuela } \\
\text { motora es más eficiente } \\
\text { realizar ejercicios pasivos } \\
\text { cada } 4 \text { hrs o cada } 8 \text { hrs para } \\
\text { prevenir la atrofia muscular } \\
\text { en un año? }\end{array}$ & $\begin{array}{c}\text { ¿En pacientes en ventilación } \\
\text { mecánica por más de } 72 \text { horas, es } \\
\text { más eficiente el tubo } \\
\text { endotraqueal con aspiración } \\
\text { subglótica que el tubo } \\
\text { endotraqueal standard con } \\
\text { aspiración de secreciones SOS } \\
\text { para la prevención de neumonía } \\
\text { nosocomial en } 1000 \text { días de uso } \\
\text { de ventilación mecánica? }\end{array}$ \\
\hline
\end{tabular}

Cuantitativa: Mide fenómenos por medio de números en un proceso deductivo y analiza a través de métodos estadísticos.

Cualitativa: Explora fenómenos en profundidad, en un proceso inductivo. Extrae los significados a contar de los datos.

Mixta: Combinación de enfoque cuantitativo y cualitativo.

En enfermería, al investigar fenómenos y respuestas humanas, el enfoque cualitativo permite explorar estos fenómenos en profundidad ${ }^{(9)}$. Pero también nuestra disciplina necesita la medición numérica y posibilidad de repetición que nos brinda el diseño cuantitativo. Un ejemplo claro son las investigaciones que permiten la medición de eventos adversos en hospitales, coberturas de vacunación, etc. Por ello, varios autores señalan la importancia del enfoque mixto que permite medir $y$ explicar fenómenos al mismo tiempo $(10,11,12)$. 
Keppes M.S., Pacheco C.

Enfoque Cuantitativo: Diseños de Estudios y Niveles de Evidencia

Entendiendo que el tipo de diseño, permite sacar conclusiones con respecto al nivel de seguridad de los resultados que describirían a una población, se establecen asociaciones que bien pueden determinar cómo se comporta una intervención. No todos los diseños de investigación tienen la misma jerarquía, a la hora de servir como evidencia para la toma de decisiones en la práctica clínica ${ }^{(13)}$. Es relevante la metodología como factor crucial que debe seguirse en forma rigurosa de acuerdo al diseño escogido.

Tipos de diseño de Estudios Epidemiológicos y Jerarquía de la Evidencia:

\section{1.- Según la finalidad del estudio}

Descriptivos: estudios que "muestran" o describen una población. Responden a la pregunta del investigador acerca de "cómo es" o "cómo está" una población o variable ${ }^{(14)}$. Son la base para otro tipo de estudios como los analíticos o los experimentales.

Analíticos: Son estudios que responden a porqué sucede un fenómeno. Muestran cómo se asocia una variable o factor de riesgo con una enfermedad, o condición de salud.

Experimentales: Son estudios donde el investigador es quien manipula la intervención para comparar dos o más grupos. Permiten medir causalidad y asociación, con seguridad ${ }^{(15)}$. Dentro de los estudios experimentales encontramos los ensayos clínicos.

\section{Estudios Descriptivos}

-Estudios ecológicos o poblacionales; datos agregados de toda la población, la unidad de análisis es un grupo de personas. Describen la enfermedad en la población en relación a variables de interés como puede ser la edad, consumo de alcohol, alimentación, tabaco, ubicación geográfica.

Un tipo de estudio ecológico es el estudio de mapa, donde podemos asociar a ubicación geográfica, consumos, prevalencia de enfermedades etc. Proporcionan información importante cuando la variable geográfica es determinante en la condición estudiada. Un ejemplo es el estudio de Mora Alvarado, Chamizo García, y Mata Solano (2006) donde se explora la incidencia de Cáncer gástrico en Costa rica en relación con el contenido de nitritos en el agua consumida $\left({ }^{16)}\right.$.

-Series de casos: Se realiza en base a grupo de pacientes con un diagnóstico similar. Este tipo de estudios puede permitir plantear una hipótesis, pero no puede probarla. Es muy útil para el estudio de brotes epidémicos de enfermedades o el estudio de condiciones o factores de riesgo poco comunes, o tratamientos alternativos en un grupo de personas. Un ejemplo es el estudio de Mármol, Luque, Farouk, y Fernández-Crehuet (2015). En este estudio se describen las características de 261 pacientes con trastorno delirante. Se estudian variables como edad, sexo, demora en atención con los primeros síntomas etc. ${ }^{(17)}$.

-Estudios corte transversales o de prevalencia: estudian simultáneamente la 
¿Es la Revisión Sistemática el mejor estudio...?

exposición y la enfermedad en una población bien definida, en un momento determinado (cohorte). Esta medición simultánea no permite conocer la secuencia temporal de los acontecimientos. Es de gran utilidad para valorar el estado de salud de una comunidad y determinar sus necesidades. Así como los estudios descriptivos son útiles para formular hipótesis etiológicas, este tipo de estudios son usados para, entre otros objetivos, medir la sensibilidad de los sistemas de vigilancia de Infecciones asociadas a la atención en salud (IAAS). Así permiten medir prevalencia de IAAS, prevalencia de procedimientos invasivos etc. $^{(18)}$.

\section{Estudios analíticos}

Estudio de casos y controles: es muy utilizado en la investigación y se le podría describir como un procedimiento epidemiológico analítico, no experimental con un sentido retrospectivo, ya que, partiendo del efecto, se estudian sus antecedentes, en el que se seleccionan dos grupos de sujetos $^{(19)}$. Los casos son los pacientes que han desarrollado una enfermedad, los controles sujetos de características similares a los casos pero libres de enfermedad. A contar de ello se observa en los dos grupos una determinada exposición a un factor de riesgo.

Los beneficios de este tipo de estudios es que son menos costosos que los estudios de cohorte, son muy útiles para enfermedades de baja ocurrencia, en general son de corta duración.

En este tipo de estudios se suelen presentar los resultados con Odds Ratio
(OR). Esta medida de efecto no tiene una traducción literal al español, pero una aproximación es la probabilidad de que un evento ocurra y de que no ocurra ${ }^{(20)}$.

$\mathrm{Si}$ el $\mathrm{OR}=1$ no hay evidencia de asociación entre el factor de riesgo y la enfermedad. Si es mayor de 1, el factor estudiado es un factor de riesgo. Si el OR es menor de 1 , el factor estudiado es un factor de protección.

\section{Estudio de cohortes (o de seguimiento)}

En este tipo de estudio los individuos son identificados en función de la presencia o ausencia de exposición a un determinado factor. En este momento todos están libres de la enfermedad de interés y son seguidos durante un período de tiempo para observar la frecuencia de aparición del fenómeno que nos interesa. $\mathrm{Si}$ al finalizar el período de observación la incidencia de la enfermedad es mayor en el grupo de expuestos, podremos concluir que existe una asociación estadística entre la exposición a la variable y la incidencia de la enfermedad, esto se llama $\mathrm{RR}=$ riesgo relativo. Para analizar el riesgo relativo debemos tener en cuenta si es $=1$ significa que no hay asociación entre el factor de riesgo y la enfermedad (para presentar la enfermedad es igual si el paciente tiene o no tiene el factor de riesgo). Si el RR es menor de $1=$ quiere decir que el factor de riego se asocia con la enfermedad (los pacientes que tienen el factor de riesgo tienen más riesgo de presentar la enfermedad que los que no lo tienen). Si el RR es mayor de $1=$ quiere decir que el factor estudiado es un factor protector. Los pacientes que tienen este factor tienen menos riesgo de presentar la enfermedad que los que no lo tienen. 
Keppes M.S., Pacheco C.

En las medidas de efecto es importante notar la importancia del intervalo de confianza. Así en la Figura 3 se puede apreciar que el estudio A, muestra un factor protector ya que el intervalo de confianza (IC) pasa por un resultado menor de 1 . El estudio B su resultado es no significativo ya que a pesar que el inicio del intervalo es menor de 1 , pasa por el 1. En el estudio $\mathrm{C}$ el factor estudiado es un factor de riesgo ya que el inicio y el término del IC son mayores de 1. E estudio D es no significativo ya que a pesar que la mayoría del intervalo es mayor de 1 , el inicio es menor de 1 y el intervalo pasa por el 1 que es efecto nulo.

Figura 3. Medidas de efecto y su valor.

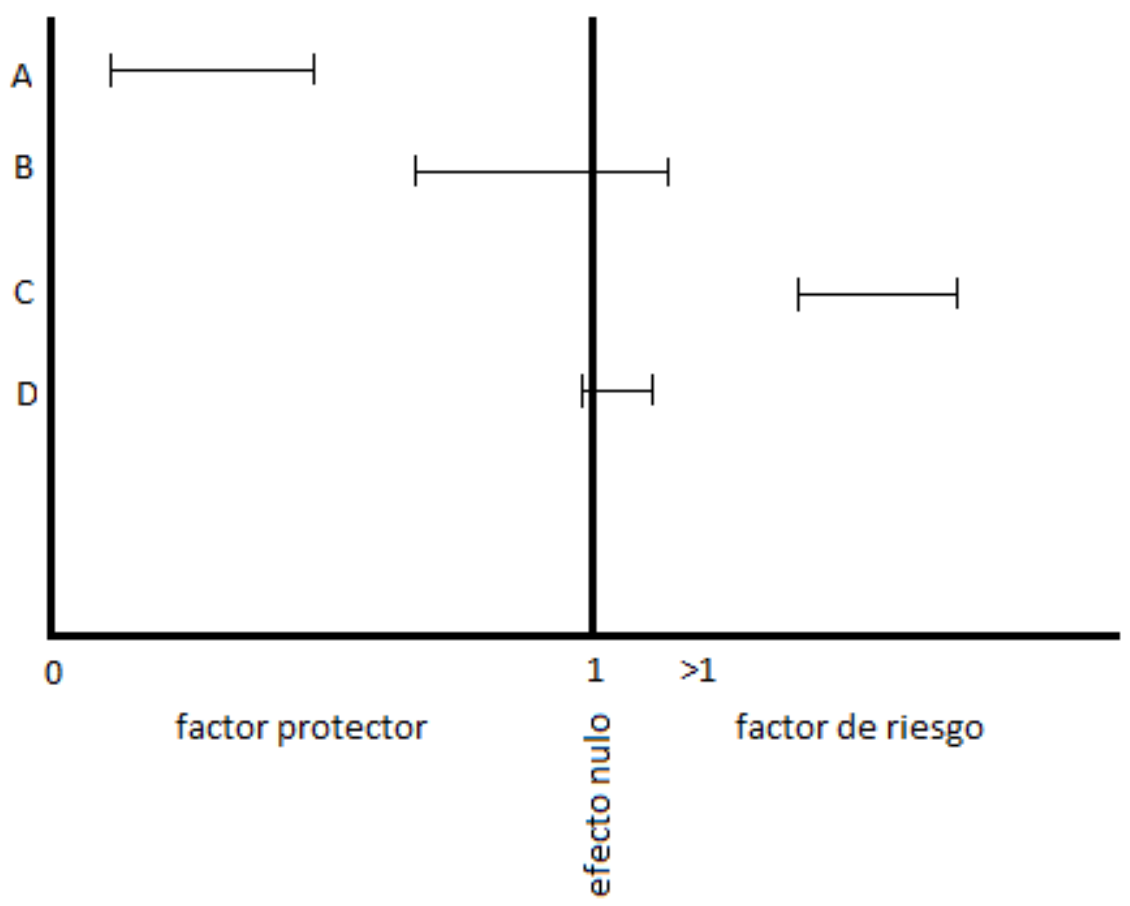

2.- Según la secuencia temporal del estudio

Los estudios se pueden clasificar según la secuencia temporal de los hechos cuando el tiempo en que ocurren los hechos es relevante para la investigación.

Estudios transversales: Son aquellos en que todas las variables son medidas al mismo tiempo. Constituye una "foto" del momento en que se miden las variables.
Por ello, si el estudio incluye posibilidad de que la medición de las variables tengan cambios estacionales (ejemplo enfermedades respiratorias que tienen cambios estacionales, o la medición de cargas laborales en períodos de vacaciones) no es el mejor diseño para esa investigación. Un estudio transversal puede ser el que aplica un instrumento para medir todas las variables en un solo momento y a contar de ello se analizan los resultados. El estudio de Moreno-Cubillos, 
et al 2007 representa un estudio transversal al medir la frecuencia de la violencia sexual contra estudiantes de programas presenciales de pregrado de la Universidad de Caldas por medio de encuestas anónimas y voluntarias ${ }^{(21)}$.

Estudios longitudinales: Son aquellos donde el tiempo es importante para la determinación de asociación entre las variables o bien en cómo se describen las variables en el estudio de Martos-Benítez, Gutiérrez-Noyola, Echeverría-Víctores (2016) donde se estudian pacientes quirúrgicos de cáncer de tórax y digestivo y se evalúa con un instrumento las complicaciones postoperatorias y su incidencia en la mortalidad y estadía hospitalaria ${ }^{(22)}$.

\section{3.- Según la asignación de factores de} estudio

Observacional: El investigador sólo observa las variables, sin manipularlas.

Experimental: El investigador manipula la intervención, estableciendo dos o más grupos para comparación.

\section{4.- Según el inicio de los hechos en relación a la cronología de los hechos}

Prospectivo: En este estudio los hechos se van registrando a medida que ocurren, desde el presente al futuro. Por ello, los estudios en que a partir de un factor de riesgo observan la aparición de un daño, son prospectivos. Un ejemplo son las cohortes. El estudio de Danilla et al. (2016) describe los cambios de calidad de vida en mujeres que fueron sometidas a cirugía de aumento mamario. En este estudio a contar de la cirugía se analiza en el tiempo (prospectivamente) que cambios se producen en las mujeres y cómo influye en su calidad de vida ${ }^{(23)}$.

Retrospectivo: Este estudio busca el registro de datos sucedidos en el pasado. Los estudios de casos y controles clásicamente son retrospectivos ya que a contar de un daño o enfermedad buscan la exposición del sujeto a un determinado factor.

\section{5.- Según el ámbito del estudio ${ }^{(24,25)}$}

Tratamiento: En estos estudios se prueba un tratamiento y su efectividad. Ejemplos de ellos son los estudios para prueba de vacunas.

Pruebas diagnósticas: son los estudios que permiten medir la sensibilidad de pruebas, como la citología exfoliativa para detección de cáncer cérvico- uterino.

Pronóstico: Estudios de riesgo de complicación de enfermedades, progresión de enfermedades clasificadas por etapas etc.

Prevalencia: Prevalencia de enfermedades o de factores de riesgo en una determinada población de estudio.

Costos: Estudios que permiten determinar costos de prestaciones de salud. El estudio de Kappes y Riquelme (2018) determina el sobrecosto de la infección de tracto urinario para pacientes con catéter urinario permanente $^{(26)}$. Este estudio de casos y controles determina que en pacientes que tienen catéter urinario permanente y desarrollan ITU el costo se eleva en 4 veces al comparar con pacientes con catéter urinario permanente pero sin infección. Se costea en base a días cama, uso de antibióticos y cultivos. 
¿Es la Revisión Sistemática el mejor estudio...?

Pirámide de la jerarquía de la evidencia según el tipo de estudios

El Dr. Brian Haynes propuso una pirámide de diseños de investigación en donde se visualiza el concepto del filtro de validez, mientras menos información los sesgos son menores pues se han aplicado los filtros correspondientes ${ }^{(27)}$. $\mathrm{Al}$ descender en la pirámide los sesgos van aumentando y también la cantidad de estudios, pues mientras más abajo menos filtrados encontraremos (Figura 4).

En la punta de la pirámide se encuentra el Meta- análisis de Ensayos Clínicos Controlados y Aleatorizados y luego las Revisiones Sistemáticas ${ }^{(28)}$. Son

Figura 4. Jerarquía de la Evidencia.

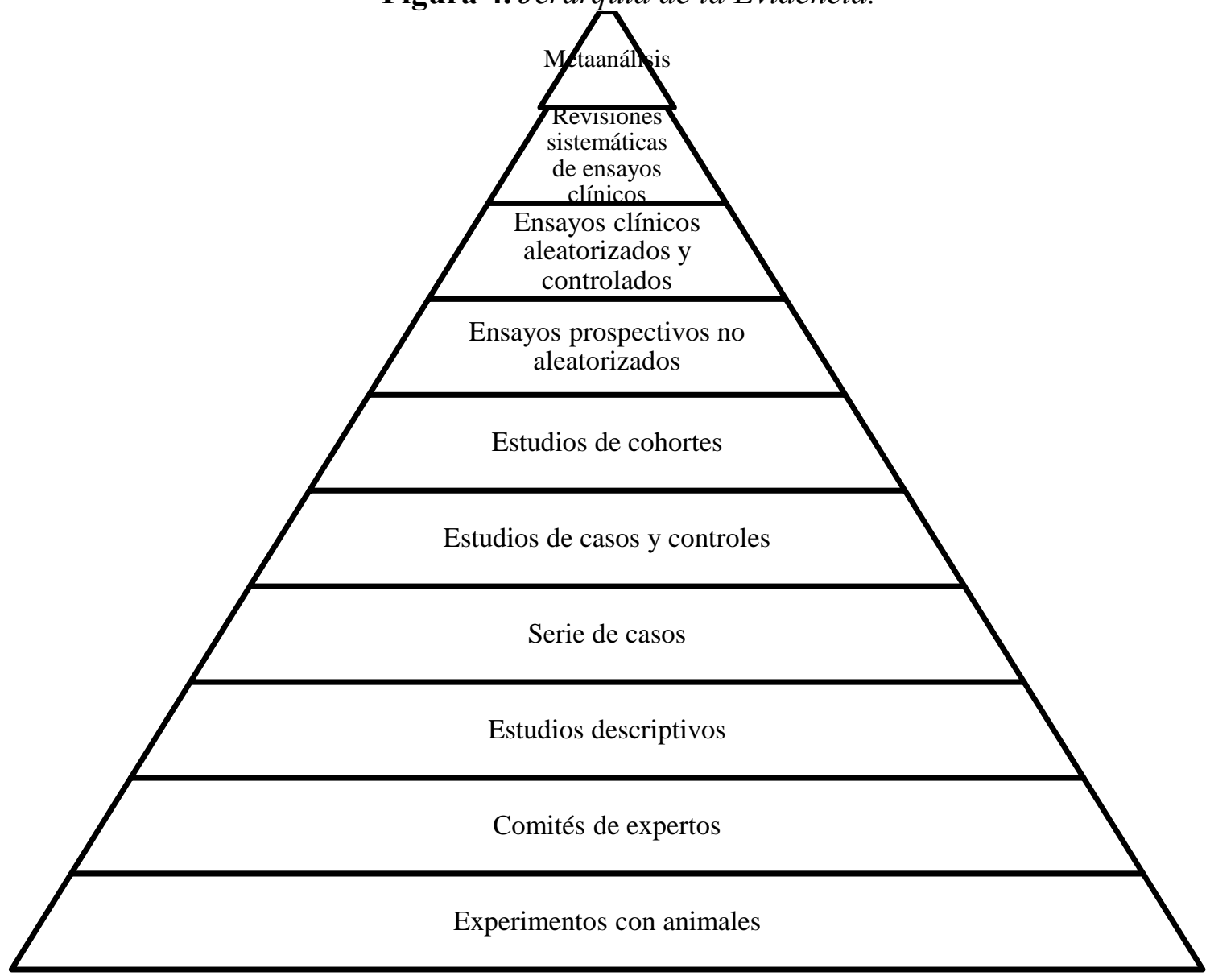

estos, por tanto, la mejor evidencia científica disponible, tanto por su rigurosa metodología como por la cantidad de información que contienen.

\section{Revisión Sistemática (RS) y Meta análisis}

Es un diseño de investigación en el que las personas son reemplazadas por los estudios o dicho de otra forma, los estudios son considerados como personas. Estos, de preferencia deben ser Ensayos Clínicos Aleatorizados Controlados.

En estos estudios el grupo revisor intenta dar respuesta a una pregunta concreta. Los estudios que den respuesta a esta pregunta, entregarán las pruebas o evidencia que ayudará al profesional a tomar la mejor decisión clínica para el paciente.

Por lo tanto, este proceso que comienza con la "formulación de la 
Keppes M.S., Pacheco C.

pregunta" debe partir con la búsqueda de información acerca de cada uno de los elementos considerados: la población o problema, la intervención propuesta y los resultados (outcome) esperados. Esta acción determinará los criterios de elegibilidad, para luego planificar con rigurosidad la metodología a seguir. La búsqueda de información debe exhaustiva y abarcar toda la evidencia disponible, publicada o no (literatura gris) considerando cuidadosamente los criterios de inclusión para que los resultados obtenidos sean lo más acotados posible con respecto a la pregunta planteada. De esta forma, los resultados obtenidos surgirán a partir de una combinación múltiple de conceptos y términos relacionados directamente con la investigación o con el paciente.

Las estrategias elaboradas, deben correrse en diferentes bases de datos para minimizar sesgos y no omitir resultados, siendo Medline, Embase, Cochrane Library y la Biblioteca Virtual en Salud (BVS) , SCOPUS, WOS y PSYCINFO sitios obligatorios según la rama del conocimiento, pues son los que contienen gran parte de la producción científica mundial. Dada la complejidad de esta labor se recomienda la realicen documentalistas o bibliotecarios clínicos especializados en Medicina Basada en Evidencia.

En el primer tamizaje de los estudios recopilados, se seleccionarán los que cumplan con los criterios definidos por el Grupo Revisor. En esta etapa de la investigación, cumplen un rol fundamental los documentalistas clínicos, que cuentan con los conocimientos adecuados para realizar búsquedas a través de bases de datos y recuperar los artículos, pues se debe tener experiencia en las diferentes interfaces de cada una de ellas y en lo posible una red de apoyo en lo que a información se refiere.

Los datos obtenidos desde los estudios seleccionados pueden, a través de test estadísticos llamados pruebas de heterogeneidad, resumirse en un metaanálisis y así obtener indicadores únicos, que llevarán a robustecer su validez. Si los datos no son homogéneos se recomienda hacer una RS cualitativa sin metaanálisis ${ }^{(29)}$.

\section{Ensayo clínico aleatorizado controlado}

Este diseño de estudio experimental, se trata de una exposición determinada de un grupo de individuos, que sería el "grupo de intervención" y se compara con otro grupo con las mismas características, que sería el "grupo de control", el que no estaría expuesto a lo mismo, puede ser a un placebo $u$ otra exposición diferente. Por lo tanto, es el investigador el que manipula la intervención.

Para su confiabilidad, en este tipo de estudios experimentales se asigna en forma ciega y aleatoria a los participantes en 2 o más grupos de iguales características, su participación estará a cargo del grupo investigador, el que deberá estar ciego a dicha asignación e ir controlando, evaluando y comparando los resultados obtenidos de ambos grupos.

No puede existir manipulación de ningún tipo a fin de robustecer la evidencia de la eficacia o no eficacia de la intervención propuesta. Los resultados y conclusiones serán obtenidos de la comparación de los desenlaces de ambos $\operatorname{grupos}^{(30)}$. 
¿Es la Revisión Sistemática el mejor estudio...?

Las intervenciones pueden ser variadas, por ejemplo, en el campo de la Enfermería podrían referirse a algún cuidado del paciente, como por ejemplo la frecuencia del aseo bucal, el uso de dispositivos o insumos para prevención de úlceras por presión, o bien para medir un desenlace importante asociado (ejemplo, incidencia de Neumonía asociada a Ventilación mecánica) ${ }^{(31,32,33)}$. También deben ser descritas las pruebas y escalas de valoración usadas para la detección de eventos, tales como caídas, UPP, satisfacción, etc. $^{(34,35)}$.

\section{Revisiones sistemáticas y Enfermería}

De acuerdo a lo expuesto anteriormente se deduce, que la revisión sistemática es un gran aporte para las disciplinas médicas y la Enfermería, abarcando no sólo temas biomédicos sino también de interés de la disciplina de enfermería y como profesión ${ }^{(36)}$. Se han incluido algunas Revisiones Sistemáticas Cochrane que han entregado información relevante a la práctica clínica por la validez científica alcanzada a través de su rigurosa metodología. La robustez de la evidencia apoya a la toma de decisiones y hace posible que los cuidados hacia los pacientes sean más seguros y eficaces.

Algunos ejemplos de esta afirmación serían las siguientes Revisiones Sistemáticas Cochrane:

1) Taylor, RS. Dalal, H. Jolly, K. Moxaham, T. Zawada, A. Homebased versus centre-based cardiac rehabilitation. 2010. Cochrane DatabaseSyst Rev. Jan 20; ${ }^{(1)}$

Esta revisión sistemática se refiere a la comparación de dos modelos de rehabilitación cardiaca: compara un programa de rehabilitación cardiovascular realizado en hospitales, con un programa realizado en el hogar de los pacientes. La conclusión muestra que las intervenciones en el hogar y en el hospital son similares en cuanto a sus beneficios y calidad de vida y en algunos casos, los pacientes muestran tener mejor adherencia al tratamiento. Si bien se necesita mayor evidencia para hacer afirmaciones categóricas, se visualiza a largo plazo la posibilidad de descongestionar los hospitales y dar relevancia a los cuidados de la familia en la rehabilitación de los pacientes, etc. ${ }^{(37)}$.

2) Hulzebos EH, Smit Y, Helders PP, van Meeteren NL. Preoperative physical therapy for elective cardiac surgery patients.2012. Cochrane DatabaseSyst Rev. Nov 14;11

Revisión que se refiere a la eficacia y seguridad de una fisioterapia preoperatoria en pacientes de cirugía cardiaca electiva. Se revisan los resultados de ocho estudios con la participación de 856 pacientes. Los resultados muestran que la fisioterapia respiratoria reduce el número de pacientes que experimenta atelectasias y neumonía en el postoperatorio y menos días de hospitalización $^{(38)}$.

3) Webster, J. Osborne, S. Rickard, C. New, K. Clinically-indicated replacement versus routine replacement of peripheral venous catheters.2015.Cochrane Database of Systematic Reviews 2015, Issue 8. Art. No.: CD007798.

Esta revisión es acerca del reemplazo del catéter venoso periférico cuando se indica clínicamente versus reemplazo sistemático. El procedimiento puede provocar un malestar considerable a 
Keppes M.S., Pacheco C.

los pacientes y es de alto costo para los servicios públicos por lo frecuente de su instalación.

La revisión no encontró pruebas definitivas de beneficio del cambio de los catéteres cada 72 o 96 horas como es la indicación. Esta conclusión da la posibilidad de cambiar la política y los catéteres podrían reemplazarse sólo con indicación clínica. Este hecho ahorraría el dolor innecesario a los pacientes y disminuiría en forma considerable el costo asociado, aportando un ahorro significativo a la salud pública al ser un procedimiento común en hospitales ${ }^{(39)}$.

Estas revisiones, son un ejemplo de cómo se puede cambiar y mejorarla práctica clínica en Enfermería basándose en evidencia confiable, preguntas clínicas concretas y evidencia científica. Muchas de las intervenciones que se realizan hasta el día de hoy, aún obedecen a evidencia obsoleta $^{(40)}$.

Luego de la información revisada se puede concluir que el enfoque de investigación mixto es un importante aporte a la práctica de enfermería. Dentro del enfoque cuantitativo, las revisiones sistemáticas aportan evidencia confiable para basar la práctica de enfermería.

Por último, las enfermeras clínicas, médicos, kinesiólogos y otros profesionales de la salud pueden a través del perfeccionamiento y la educación continua, ser agentes de cambio, revisando críticamente sus actividades diarias y buscando evidencia científica confiable, para mejorar la eficacia, seguridad y calidad de los procedimientos que realizan en el cuidado de los pacientes.

\section{REFERENCIAS BIBLIOGRÁFICAS}

1. Grove, S. Gray, J. Burns, N. Investigación en enfermería. Desarrollo de la práctica basada en la evidencia. 6ta Ed. Elsevier.2016. 544 p.

2. Munley R, Rowell P. Claiming the future of nursing through nursingsensitive quality indicators. Nurse Adm Q 2003;27 (4): 273-284.

3. Hernández L. Metodología de la investigación en Ciencias de la Salud. $3^{\mathrm{a}}$ ed. Bogotá, Colombia: Ediciones ECOE; 2012. 134 p.

4. Luengo, C. Paravic, T. Autonomía Profesional: factor clave para el ejercicio de la Enfermería Basada en la Evidencia. Index Enferm 2016; 25(12): 42-46.

5. Cañón, M. Buitrago-Gómez, Q. La pregunta de investigación en la práctica clínica: guía para formularla. Revista Colombiana de Psiquiatria. Article in Press $2016 \quad$ DOI https://doi.org/10.1016/j.rcp.2016.06.0 04

6. Vera, O. El aprendizaje basado en problemas y la medicina basada en evidencias en la formación médica. Rev. Méd. La Paz 2016. 22(2): 78-86.

7. Bottaro F. ¿Cómo leer la literatura científica? Hematología 2013;17 (1): 79-85.

8. Hernández, R. Fernandez, C. Baptista, P. Metodología de la Investigación. 6ta Edición. México DF: Mc Graw Hill, 2014. 3-8p.

9. León, J. La investigación cualitativa y el desarrollo profesional de enfermería. Cuid salud. 2015; 2(2):220-234.

10. Simons, P. Variabilidad y calidad de la práctica clínica de enfermería en pacientes con Diabetes mellitus, 
¿Es la Revisión Sistemática el mejor estudio...?

hospitalizados Revista Ciencia y Tecnología 2017. 20(1):67-89.

11. Katz, J. Vandermause, R. McPherson, S. Barbosa-Leiker, C. A demonstration of mixed-methods research in the health sciences. Nurse Res. 2016. 18;24(2):24-29.

12. Ochieng, B. Meetoo, D. Using mixed methods when researching communities. Nurse Res. 2015. (1):16-19.

13. Ochoa, C. Evidencia y recomendación. Rev. ORL, 2016 7( 2): 67-71

14. Argimon, J. Jiménez, J. Métodos de investigación clínica y peidemiológica +StudentConsult en español. 4ta Ed. Elsevier 2012. 416 p.

15. Lazcano-Ponce E, Salazar-Martínez E, Hernández-Ávila M. Estudios epidemiológicos de casos y controles: Fundamento teórico, variantes y aplicaciones. Rev cubana Hig Epidemiol 2007.45 (3) Disponible en: http://scielo.sld.cu/scielo.php?script=s ci_arttext\&pid=S156130032007000300009\&lng=es.

16. Mora, DA. Chamizo, H. Mata, A. Estudio exploratorio sobre la incidencia de cáncer gástrico y los contenidos de nitratos en el agua potable en Costa Rica. RevCostarric Salud Pública. 2006;15 (28): 17-28. [Consultado el 11 de Diciembre de 2017].

17. Mármol F, Luque R, Farouk M, Fernández-Crehuet R. Estudio descriptivo de series de casos de trastorno delirante. Rev Chil Neuropsiquiatr 2015;53 (4): 241-50. [Consultado el 11 de Diciembre de 2017].

18. Valdés, LE. Leyva, T. Prevalencia de infecciones asociadas a la asistencia sanitaria en hospitales provinciales de Santiago de Cuba. MEDISAN 2013;17
(12): 9131-9143. [Consultado el 11 Diciembre de 2017].

19. Parreño, A. Metodología de investigación en salud. 1Ed. Espoch. Ecuador, 2016. 126 p.

20. Cerda J, Vera C, Rada G. Odds ratio: aspectos teóricos y prácticos. RevMed Chile 2013;141 (10): 13291335. [Consultado el 11 Diciembre de 2017].

21. Moreno-Cubillos CL, Osorio-Gómez LS, Sepúlveda-Gallego LE. Violencia sexual contra las estudiantes de la Universidad de Caldas (Colombia): estudio de corte transversal. Rev ColombObstetGinecol 2007;58 (2): 116-123. [Consultado el 11 Diciembre de 2017].

22. Martos- Benitez, D. Gutiérrz-Noyola, A. Echeverría- Víctores A. Complicaciones postoperatorias $\mathrm{y}$ resultados clínicos en pacientes operados por cáncer torácico y gastrointestinal: Estudio de cohorte prospectivo. RevBras Ter Intensiva. 2016;28(1):40-48

23. Danilla S, Ríos MA, Cuevas P, Troncoso E, Domínguez C, Jara R, et al. Cambios en la calidad de vida en mujeres sometidas a aumento mamario. Resultados preliminares de un estudio de cohortes. RevChilCir 2016;68 (4): 289-294.

24. Manterola C, Zavando D. Cómo interpretar los "Niveles de Evidencia" en los diferentes escenarios clínicos. RevChilCir 2009;61 (6): 582-595. Disponible en: http://www.scielo.cl/scielo.php?script= sci_arttext\&pid=S071840262009000600017\&lng=es [Consultado el 18 Junio de 2017]. 
Keppes M.S., Pacheco C.

25. Fletcher R, Fletcher S. Epidemiología Clínica. 5 Ed.Lippincott Williams and Wilkins Wolters Kluwer Health. 2016. 272 p.

26. Kappes M, Riquelme V. Urinary Tract Infection: Cost Study. Hosp Top 2018 Article in Press DOIhttps://doi.org/10.1080/00185868. 2017.1406288

27. Karlsson J, Marx R, Nakamura N, Bahandari M. A Practical Guide to Research: Design, Execution, and Publication. Arthroscopy 2011;278 (4):1-10.

28. Manterola C, Asenjo-Lobos C, Otzen T. Jerarquización de la evidencia. Niveles de evidencia y grados de recomendación de uso actual. Rev Chilena Infectol 2014;31 (6): 705-718.

29. Hutton, B. Catalá-López, F. Moher, D. La extensión de la declaración PRISMA para revisiones sistemáticas que incorporan metaanálisis en red: PRISMA-NMA. MedClin (Barc). 2016.

http://dx.doi.org/10.1016/j.medcli.201 6.02 .025

30. Cuevas, O. Molina, A. Fernández, D. Los ensayos clínicos y su impacto en la sociedad. Medisur .2016 ; 14( 1 ): 1321.

31. Manriquez J, Valdivia G, Rada G, Letelier L. Análisis crítico de ensayos clínicos randomizados publicados en revistas biomédicas chilenas. RevMed Chile 2005; 133 (4): 439-446.

32. Carvajal C, Pobo A, Diaz E, Lisboa T, Llauradó M, Rello J. Higiene oral con clorhexidina para la prevención de neumonía en pacientes intubados: revisión sistemática de ensayos clínicos aleatorizados. MedClin 2010;135 (11): 491-97.
33. Jarillo A. Estudio aleatorizado sobre la eficacia de los ácidos grasos hiperoxigenados (AGHO) en la prevención de lesiones cutáneas. Reduca 2010;2 (1): 607-628.

34. Parra DI, Camargo-Figuera FA, Rey Gómez R. Eventos adversos derivados del cuidado de enfermería: flebitis, úlceras por presión y caídas. EnfermGlob 2012;11 (28): 159-169.

35. Barrientos-Sánchez J, HernándezCantoral A, Hernández-Zavala M. Adaptación y validación de un instrumento de valoración de riesgo de caída en pacientes pediátricos hospitalizados. EnfermUniv 2013;10 (4): 114-119.

36. Canales-Vergara M., Valenzuela-Suazo S., Paravic-KlijnT.. Condiciones de trabajo de los profesionales de enfermería en Chile. Enferm. univ.2016 ; 13( 3 ): 178-186.

37. Taylor RS. Dalal H. Jolly K. Moxaham T. Zawada A. Home-based versus centre-based cardiac rehabilitation. Cochrane Database Syst Rev 2010;20 (1).

38. Hulzebos EH, Smit Y, Helders PP, van Meeteren NL. Preoperative physical therapy for elective cardiac surgery patients. Cochrane Database Syst Rev 2012;11.

39. Webster J, Osborne S, Rickard C, New K. Clinically-indicated replacement versus routine replacement of peripheral venous catheters.Cochrane DatabaseSystRev 2015; 8.

40. Morán L., Quezada Y., García A., González P., Godínez S., Aguilera M.. Resolver problemas y tomar decisiones, esencia de práctica reflexiva en enfermería. Análisis de la literatura. Enferm. univ.2016 ; 13( 1 ): 47-54. 

¿Es la Revisión Sistemática el mejor estudio...?

2018, Horiz. Enferm., 28,3,224-237 